\title{
Machinations of the marrow
}

\author{
Nadia Rosenthal \\ European Molecular Biology Laboratory, Mouse Biology Programme, Monterotondo, Rome, Italy \\ J. Clin. Invest. 111:29-30 (2003). doi:10.1172/JCI200317546.
}

All the wealth I had ran in my veins. -Shakespeare, The Merchant of Venice

The common provenance of the blood and the vessels through which it flows lies deep in the embryonic mesoderm. There, morphogenesis of embryonic vasculature begins with specification of endothelial precursor cells, or angioblasts, which associate into vascular cords, differentiate into endothelial cells, and assemble into capillaries. These remodel to form a vascular network, around which mesenchymal cells are then recruited to form the vascular wall and stimulated to differentiate into smooth-muscle. Creating new blood vessels in the adult, long after the initial vascular system has been laid down, is necessarily a complex affair, and it is not clear how to recapitulate the original developmental program in cases where tissue regeneration depends upon extensive neovascularization.

Enter stem cells. In the embryo, the endothelial lining of certain blood vessels has been proven a rich source of multipotent stem cells that give rise to hematopoietic precursors. These later convene in the bone marrow, which becomes the chief site of hematopoiesis. But where do vascular progenitors hide? Although the constant requirement for new blood vessels in regenerating tissue would seem to call

\footnotetext{
Address correspondence to: Nadia Rosenthal, European Molecular Biology Laboratory Mouse Biology Programme, via Ramarini 32, 00016 Monterotondo, Rome, Italy. Phone: 39-06-90091-241;

Fax: 39-06-90091-272;

E-mail: rosenthal@embl-monterotondo.it. Conflict of interest: The author has declared that no conflict of interest exists.

Nonstandard abbreviations used: side population (SP).
}

for local stem cell pools, marked numbers of peripheral blood and bone marrow cells have been found in regenerating vasculature (1-3). Exogenous bone marrow-derived endothelial progenitors can also contribute to neovascularization, inducing neoangiogenesis in cases of experimental injury (4). The formation of new vasculature has been heralded as a clear example of adult stem cell plasticity, where multiple tissue types derive from an uncommitted, multipotent progenitor population.

But how plastic are the precursors and where do they originate? The enriched but impure cell populations used in many stem cell plasticity studies are heterogeneous enough to contain progenitors with diverse, restricted potentials (5). Delivered as a mix, these populations could, in principle, contribute to the reconstruction of complex structures comprising multiple tissue types without the need for transdifferentiation.

The study by Majka and colleagues in this issue of the JCI (6) elegantly illustrates this concept. These authors had previously discovered significant numbers of hematopoietic stem cells in skeletal muscle, in large part derived from circulating marrowderived cells (7). In the present study,

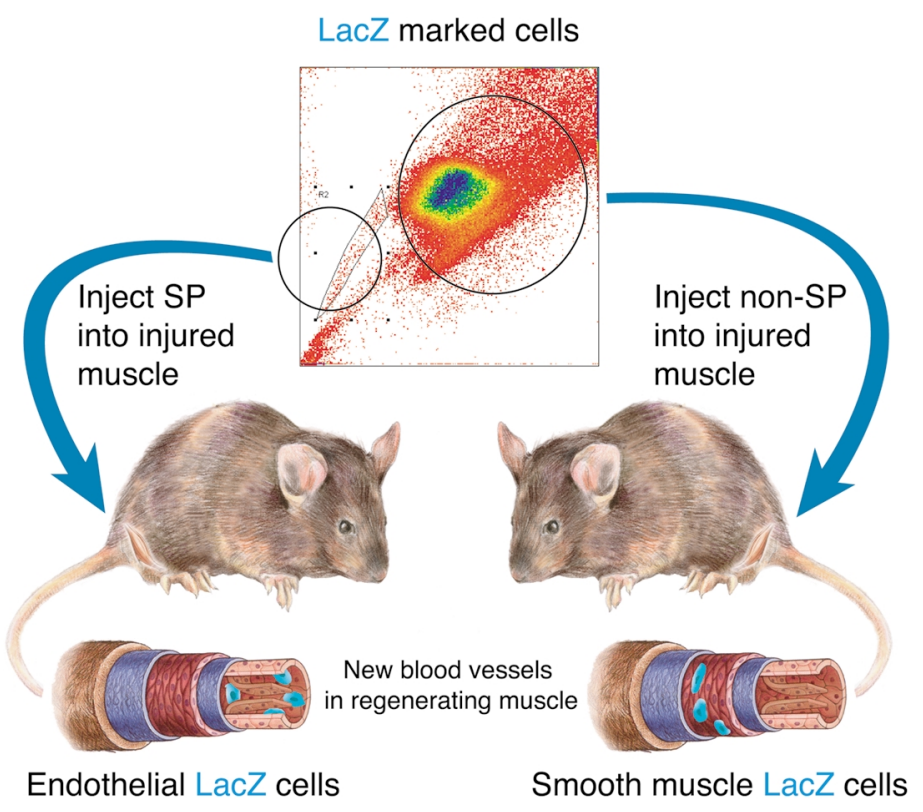

Figure 1

Patterns of engraftment by marked progenitor cells in the regenerating vasculature of injured muscle. SP and non-SP cells from lacZ-expressing Rosa26 mouse muscles were isolated by FACS analysis (upper panel) and injected into the injured muscles of genetically matched recipient mice. Engraftment of injected cells was monitored by $\beta$-galactosidase staining of recipient tissues. The SP fraction engrafted into vascular endothelium (bottom left), whereas the non-SP fraction engrafted into the vascular smooth-muscle (bottom right) of new blood vessels in the regenerating muscle. 
vascular progenitors were sought in Percoll gradient-purified cell populations from adult skeletal muscle, based on the differential efflux of a Hoechst dye. Since side populations (SPs) of cells (so called because of their appearance as a distinct population of cells during fluorescence-activated cell sorting) isolated this way can reconstitute the bone marrow of a lethally irradiated host (8), they represent the majority of hematopoietic stem cells, whereas non-SP mesenchymal cells have traditionally been considered to be less multipotent.

Isolated muscle SP cells expressed only a subset of the hematopoietic and vascular endothelial progenitor markers typical of bone marrow SPs, perhaps reflecting their more restricted potential (6). Conversely, the non-SP fraction of muscle cells expressed genes typical of smooth-muscle precursors, as well as more multipotent lineage markers. Yet both muscle cell populations could derive from the bone marrow. This was demonstrated by reconstitution of wild-type mice with bone marrow SP cells, isolated from a donor mouse carrying a ubiquitously expressed lacZ marker, which allowed the subsequent fate of the engrafted bone marrow to be traced. After 12 months, SP and non-SP populations purified from skeletal muscle of the reconstituted mice were largely lacZ-positive, indicating that the bone marrow gradually replenished resident muscle progenitor cells that had presumably been ablated by the original irradiation protocol. Although marrow readily repopulates muscle satellite cell pools in irradiated mice (9), it was not appreciated that these relocated cells contributed to the non-SP fraction.

Fortuitously, the purification of these two populations by Hoechst dye efflux in the present study uncovered a new facet of stem cell heterogeneity, by segregating two distinct building blocks of the vasculature. When SP and non-SP cells derived from lacZmarked skeletal muscle were injected into injured muscles of wild-type mice, cells in the SP fraction contributed exclusively to the endothelial component of the neovasculature after toxininduced damage, whereas those in the non-SP fraction were found exclusively in the smooth-muscle component (6). Given the arbitrary nature of the fractionation, SP and non-SP populations are likely to be far from homogenous, so the specific characteristics distinguishing the two progenitors have yet to be defined. Nevertheless these data show that cells emanating from the marrow can give rise to vascular progenitors residing in adult skeletal muscle, and that given the appropriate microenvironment and the impetus of injury, distinct subsets of these progenitors are capable of contributing to the endothelial and vascular smooth-muscle components of newly forming blood vessels.

Taken in the context of previous observations, this study by Majka et al. (6) sheds light on the diversity of adult tissue types to which cells from the marrow can contribute, albeit under circumstances of systemic stress (irradiation) and injury (muscle damage). It supports the notion that the heterogeneous lineage components in play during the ontogeny of the embryonic vasculature are compressed into the adult marrow, which can release cells into the circulation to aid the neovascularization process in damaged tissues. The marrow thus gives rise to distinct endothelial and muscular components of the vasculature, as well as to the blood that runs through it.

However exciting these findings may be for regenerative medicine, extending them to clinical application may prove challenging. What is the origin of this heterogeneity, and how is the wealth of potential in the adult bone marrow dispersed? Does the marrow provide the necessary individual components of regeneration only in the context of extreme experimental manipulations? It is still controversial to what extent marrow-derived cells can be coerced to repopulate tissues (10). It also remains to be seen how efficient this process can be made in a nonirradiated host where resident tissue stem cells have not been depleted, and how purified the different components should be for effective engraftment. The cumulative evidence points to a developmental heterogeneity elaborated in the embryo that persists in stem cell progenitors throughout the body, that perhaps derives from equally heterogeneous populations in the marrow itself, and that current sorting procedures fall far short of capturing. Until more exacting methods are devised to tease out the multitudes of progenitor cell types residing in our veins, the machinations of the marrow will continue to surprise us.

1. Shimizu, K., et al. 2001. Host bone-marrow cells are a source of donor intimal smooth-muscle-like cells in murine aortic transplant arteriopathy. Nat. Med. 7:738-741.

2. Jackson, K.A., et al. 2001. Regeneration of ischemic cardiac muscle and vascular endothelium by adult stem cells. J. Clin. Invest. 107:1395-1402.

3. Sata, M., et al. 2002. Hematopoietic stem cells differentiate into vascular cells that participate in the pathogenesis of atherosclerosis. Nat. Med. 8:403-409.

4. Kocher, A.A., et al. 2001. Neovascularization of ischemic myocardium by human bone marrowderived angioblasts prevents cardiomyocyte apoptosis, reduces remodeling and improves cardiac function. Nat. Med. 7:430-436.

5. Orkin, S.H., and Zon, L.I. 2002. Hematopoiesis and stem cells: plasticity versus developmental heterogeneity. Nat. Immunol. 3:323-328.

6. Majka, S.M., et al. 2003. Distinct progenitor populations in skeletal muscle are bone marrow derived and exhibit different cell fates during vascular regeneration. J. Clin. Invest. 111:71-79. doi:10.1172/JCI200316157

7. McKinney-Freeman, S.L., et al. 2002. Musclederived hematopoietic stem cells are hematopoietic in origin. Proc. Natl. Acad. Sci. USA 99:1341-1346.

8. Jackson, K.A., Mi, T., and Goodell, M.A. 1999. Hematopoietic potential of stem cells isolated from murine skeletal muscle. Proc. Natl. Acad. Sci. USA. 96:14482-14486.

9. LaBarge, M.A., and Blau, H.M. 2002. Biological progression from adult bone marrow to mononucleate muscle stem cell to multinucleate muscle fiber in response to injury. Cell. 111:589-601.

10. Wagers, A.J., Sherwood, R.I., Christensen, J.L., and Weissman, I.L. 2002. Little evidence for developmental plasticity of adult hematopoietic stem cells. Science. 297:2256-2259. 